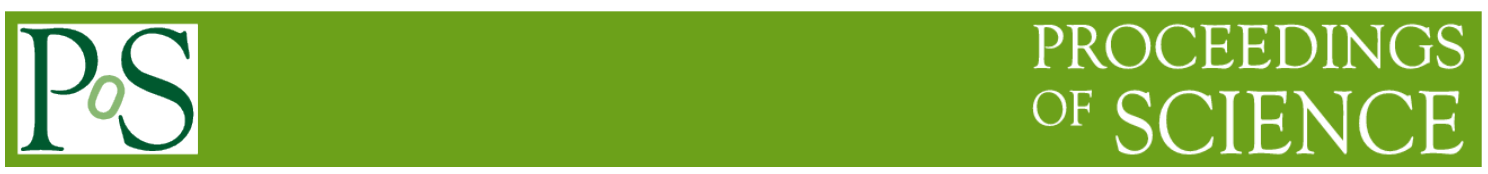

\title{
Design and Implementation of Production Management Information System for Jiujiang Railway Track Depot
}

\section{Conglin $\operatorname{Ran}^{1}$}

Information Technology Center

Jiujiang University

Jiujiang, Jiangxi, 332005, China

E-mail: rclejju.edu.cn

Upon analyzing the actual situation and the existing problems of Jiujiang railway track depot by making use of the information technology and the network technology, it puts forward to establish a production scheduling management information system solution based on the B/S mode. We design the system structure, function model and implementation using C\# ADO.NET programming technology and SQL(Structured Query Language) Server database technology. As a result, the system has been put into practice at low cost, the efficient production management and the transaction management, which has created a stable environment for the transportation of materials and passengers.

CENet2015

12-13 September 2015

Shanghai, China

${ }^{1}$ Speaker 


\section{Introduction}

The railway track depot is a special agency to manage and repair the train tracks and bridges within its jurisdiction. Jiujiang railway track depot is responsible for the railway lines of Jiujiang area. It includes 19 workshops and 93 Work areas. With the rapid development of information technology, It has been carrying out the informatization construction actively to improve its management level; thus the main information project plan for them is to build a production management information system for producing and managing the auxiliary. The system has been in use. It has reduced staff and improved the work efficiency in a unified standard.

\section{Existing Status and Problems}

It has set up a security production and scheduling command center formed by the road and bridge department, the line branches and the safety department. It consists of 19 workshops including stations, uplink and downlink. The organization structure is shown in Fig. 1. Every level has different responsibilities. There are many characteristics, such as dispersed location, complex business, arbitrarily variable operation. These problems have led to some difficulties for production and dispatch management under the condition of no information technology.

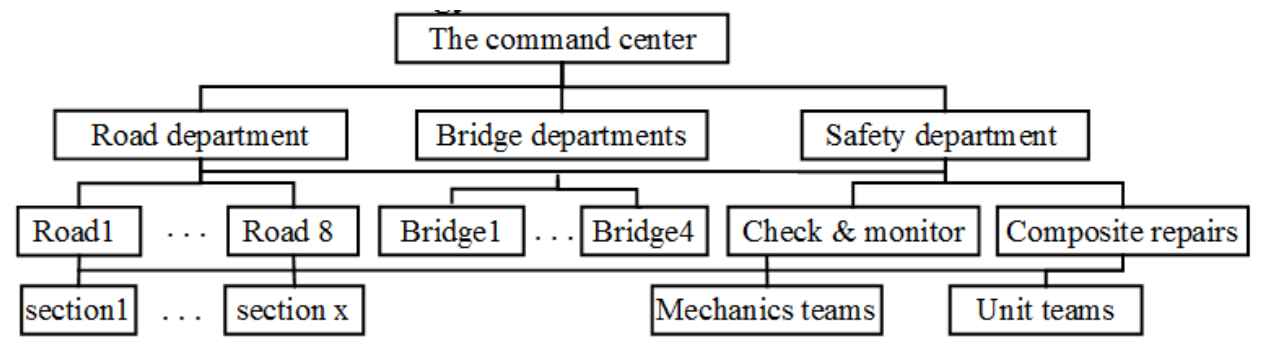

Figure 1 : Organization Structure

\subsection{Work Efficiency and Security Risks}

Over the years, it has finished the transitional information and approval of daily work by using excel or word software. No timely data report has led to the delayed examination, which has affected the normal operation and the next personnel arrangements, and even affected the routes of transportation vehicles. What the command center has grasped in respect of the operation plans information was not achieved timely, which has thus led to the occurrence of major accidents in production; and the production efficiency was extremely low.

\subsection{Limited Information Technology Level}

In addition to the permanent staff, the computer knowledge of other workers of short-term contract was very limited. The requirement on informatization construction engineering thus raises the level of computer literacy among the zones and the workshop heads so that they must adapt themselves to the development of information technology and business, which is determined by the urgency of business operation and management.

\section{Requirement Analysis}

\subsection{Main Function Requirements}

In order to solve the problems of differential data, inaccurate report data and incorrect approvals, various data are needed to be electronically managed and uniformed as per the data standards. Ensure the works can achieve global consistency and share data. It's a comprehensive 
management system, involving the staff management, the production scheduling management, the old rail materials and system management. Main functions are shown in Fig. 2.

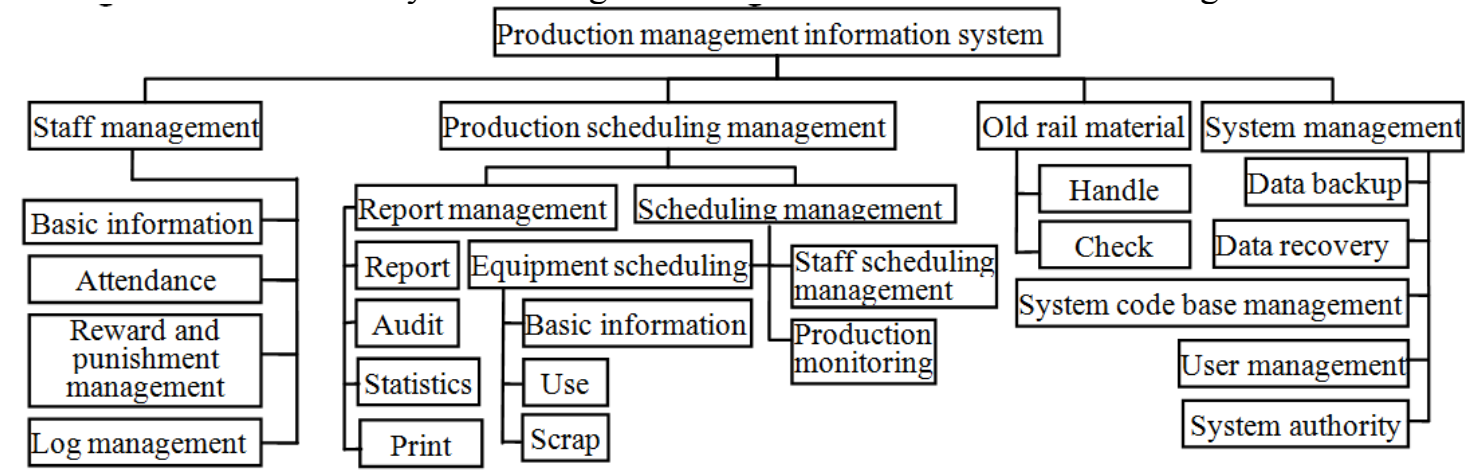

Figure 2 : Function of the System

\subsection{System Performance Requirements}

The standard and specification of the system user interface are to be unified, operated simply and to be in line with user's habit [1].

The backend menus of the system ensures the integrity of the system data. In the event of power outages or natural disasters, the system data can be quickly restored. Establishing the system operation logs is to keep a record of all users of the system operating methods for the purpose of tracking the responsibility.

As to the software development and from the users' point of view, the scalability and portability of the system should be taken into account in the early design. It's easy to be developed and expanded further. Various software development documents should be improved and saved, or the system manual should be provided in a video format so that the operators can quickly grasp the use of the system.

\section{Design}

According to the system requirements, the entire design of the system might include hardware structure, system structure and function module.

\subsection{Hardware Structure}

Each work area is located at remote place; thus the system must be built by making use of the $\mathrm{B} / \mathrm{S}$ model. All operators at different locations access the system by intranet. Each production workshop, as a client, is not required to maintain the system so as to ensure the system security [2]. The system hardware structure is shown in Fig. 3. The database server and the file server are connected by the local area network. Each workshop visits the system by the enterprise internal network [3]. 


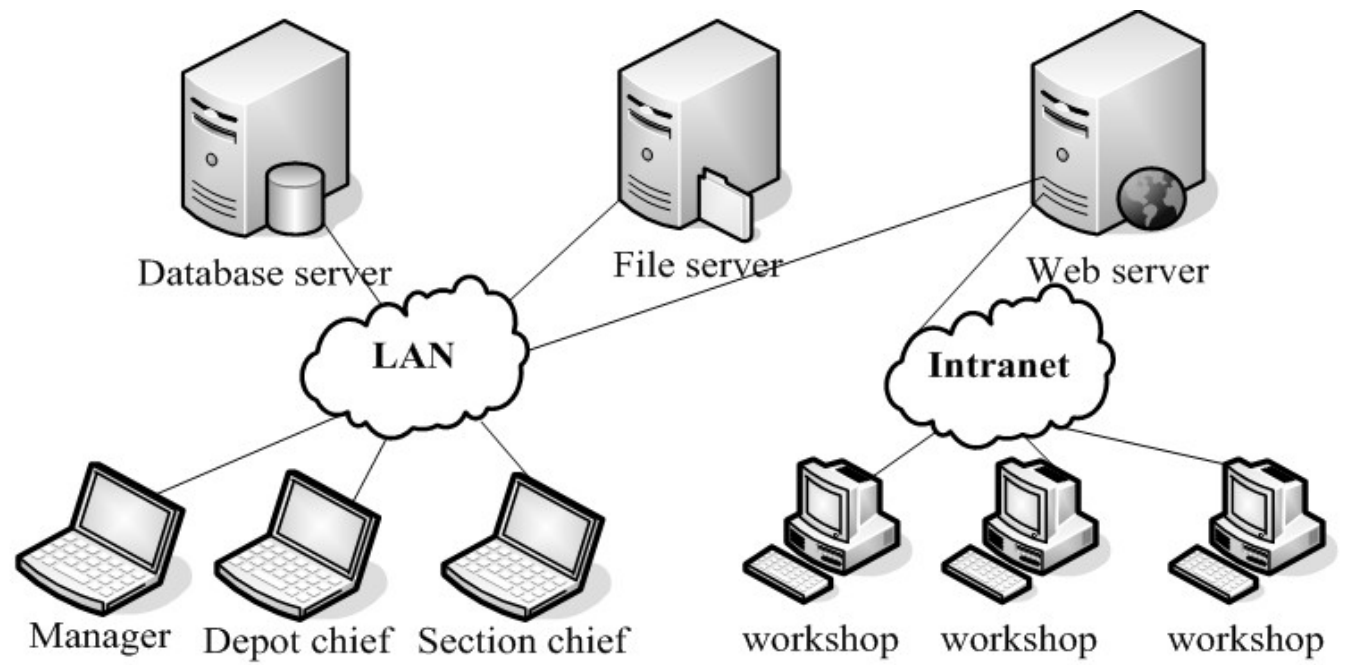

Figure 3 : System Hardware Structure

\subsection{Architecture}

It's built by three - layer structure model. It consists of the user interface layer, the web server layer(the business layer) and the data layer. The architecture is shown in Fig. 4.

The user interface layer is a logical layer. All different users login from the same entrance and access different operational jurisdictions via web browser.

The web server layer to solve the system business logic mainly includes work logs, production business management, equipment management, old railway material management, employee management and meeting management. The operation and layout of the system must be in conformity with windows users' habits [4].

The data layer is mainly responsible for handling various types of the system's report data, including the logical link data and the file resources.

Figure 1.

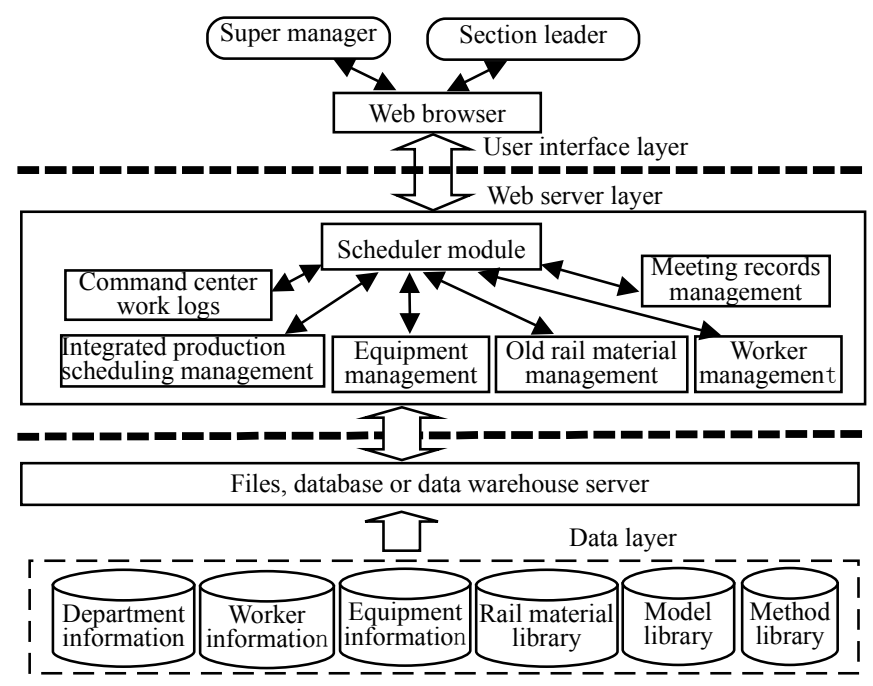

Figure 4: Architecture of the System

\subsection{Functional Modules}

Based on the actual business processes, the system process is divided into different functions and business modules; besides, it contains different basic operations, management 
information between workshop and work area, the records, the data backup and the recovery functions.

(1) The management of daily logs includes the data entry working on the train tracks, the management of tracking audit staff's operation records.

(2) The information management covers the maintenance of the productive sector, production schedules, forecast tables, feedback form, application form, integrated reports and other information management, the scheduling information and the material data on the monitoring as well as the replacement of seriously injured steel rails, blades and frogs, such as entry, reporting and audit management [5]. After the data are reported by the leaders, the information will be audited by using the two-stage audit system. The information is confirmed before corresponding production jobs. Either of the two audits is dismissed, and then the entire job scheduling scheme approval will be returned to the workshop. The specific process is shown in Fig. 5.

(3) Information management of all devices, employees, old track materials and meeting records.

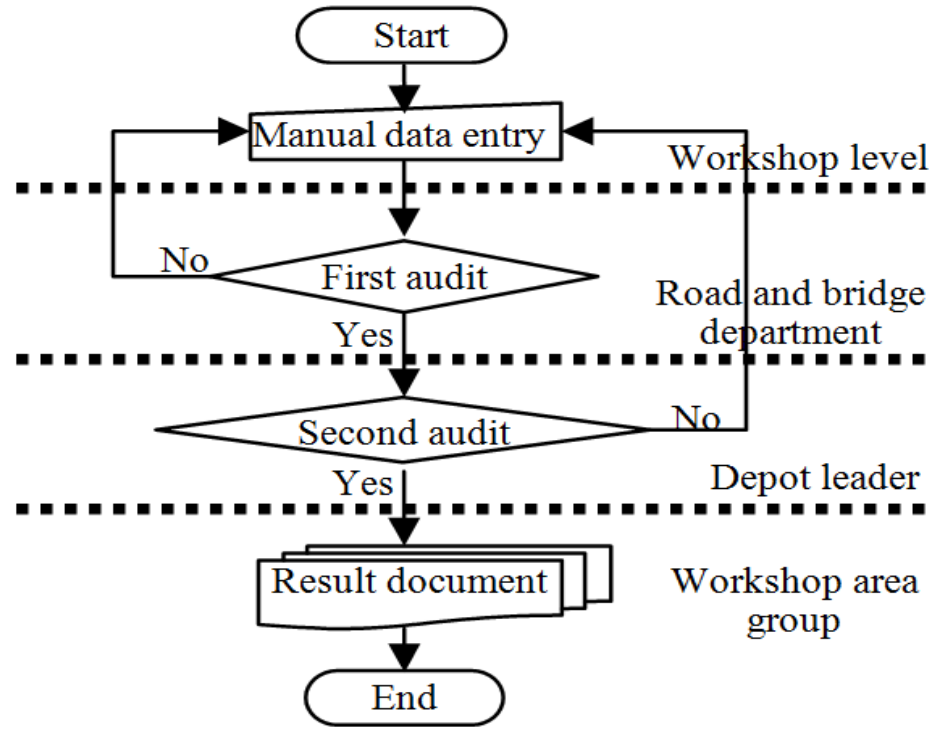

Figure 5 : Production Scheduling \& Business Audit Process

\section{Implementation}

The presentation layer of the system is developed by using HTML and JavaScript technology to select ASP.NET technology to develop the system's logical layer. The database of the system is SQL Server 2005.

\subsection{Encryption of the Users' Passwords}

The users' passwords are encrypted before they are stored in database. It is also useless for hackers to download database. When the user inputs the register information, the password data is encrypted by using md5 algorithm as stored in database. When the users login the system, the data input by such users and encrypted by using md5 will be compared with the encrypted string in the database so that the work of password verification can be accomplished.

\subsection{Data Review}

The system administrators and ordinary users enter from the same entrance. After the users have successfully logged, they only see the menu function role in their range. The production scheduling module is shown in Table 1. 


\begin{tabular}{|l|c|c|c|c|c|c|}
\hline Roles Functions & $\begin{array}{c}\text { System } \\
\text { managem }\end{array}$ & $\begin{array}{c}\text { User } \\
\text { managem }\end{array}$ & $\begin{array}{c}\text { Job data } \\
\text { entry }\end{array}$ & $\begin{array}{c}\text { Delete job } \\
\text { data }\end{array}$ & $\begin{array}{c}\text { Operation } \\
\text { audit }\end{array}$ & $\begin{array}{c}\text { Print } \\
\text { report }\end{array}$ \\
\hline System manager & $\sqrt{ }$ & $\sqrt{ }$ & $\mathrm{x}$ & $\sqrt{ }$ & $\mathrm{x}$ & $\sqrt{ }$ \\
\hline Depot leader & $\mathrm{x}$ & $\sqrt{ }$ & $\mathrm{x}$ & $\mathrm{x}$ & $\sqrt{ }$ & $\sqrt{ }$ \\
\hline Business chief & $\mathrm{x}$ & $\sqrt{ }$ & $\mathrm{x}$ & $\mathrm{x}$ & $\sqrt{ }$ & $\sqrt{ }$ \\
\hline Workshop leader & $\mathrm{x}$ & $\mathrm{x}$ & $\sqrt{ }$ & $\mathrm{x}$ & $\mathrm{x}$ & $\sqrt{ }$ \\
\hline
\end{tabular}

Table 1: Production Scheduling Module User Menu

In the stage of reviewing the next job, all kinds of users can see the audit progress. Because there are many workshops, a large number of tables about the job information are reported. The leaders audit themselves one by one so that pages are not refreshed by using Ajax partial refresh technology in an overall manner to finish the exchanging data [6]. At any time, it's convenient to view the audit records state in order.

\subsection{Establishment of Traceable Records Data of User Logs}

The system administrators can not only supervise all users but also initialize the job data and clear off last month data. In order to guarantee the system security, it's necessary to set up logs so that the visitors enter the system successfully, and the system administrators can view any operation online and discovery the behavior records of abnormal users.

\section{Application}

The system has been put into use for more than two years. Until now, it has nearly 70,000 scheduling records and 31,967 logs records. The growth trend of data record is shown in Fig. 6. The data provides an important basis for major repairs. The qualified rate is the best. In Fig. 7, about 60 people are excellent or talented in terms of professional technology.

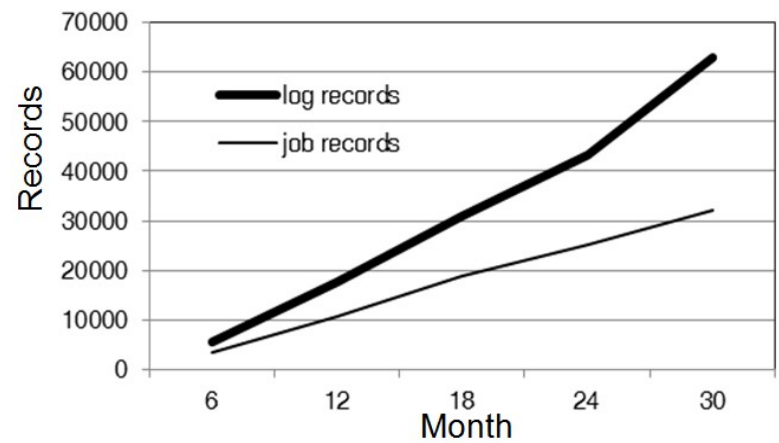

Figure 6 : Growth Trend of Database Record

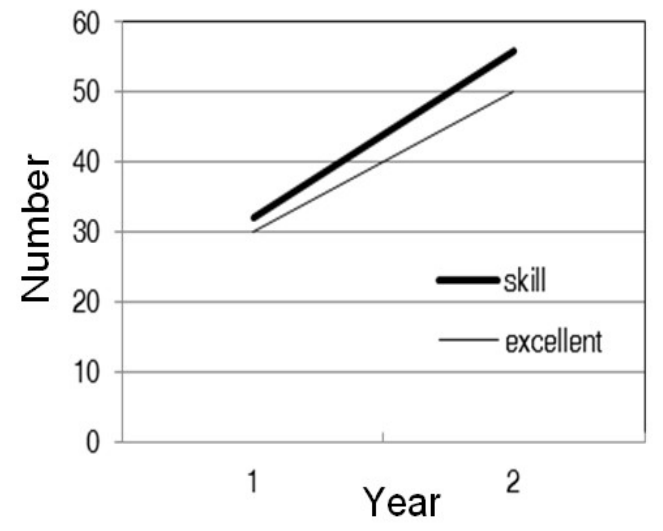

Figure 7: Number of the Talented 
The system is developed by $\mathrm{B} / \mathrm{S}$ mode and used easily. The functions are easy to be extended. Users can then visit it upon authentication at any time and from any place. It's built to solve the complexity of business data reporting and reduce the workload so that all of the problems in the production process can be maintained timely to ensure smooth train tracks and safe transport environment.

\section{Conclusion}

As the system is developed and used fully, the production scheduling management work is more standardized and systematical. It features the advantages of less investment, low environment requirements and zero maintenance; besides, it can reduce the cost and save labor. The job report is more scientific, standard and unified. It has improved the working efficiency greatly.

In short, the system can meet the practical production demanding of Jiujiang railway track depot because it ensures stable quality and features excellent ratio of bridge and tracks; furthermore, it has played a positive role in the daily production management, so it can be promoted and used in similar units.

\section{References}

[1] Xiaojing Wang, Conglin Ran. Design of manufacturing management information system in Jiujiang train crew depot[J]. Railway Transport and Economy.35(08) :38-42(2013)(In Chinese)

[2] Jingyu Zhao. Theoretical research and application of database design and normalization [J]. Eleetronic Technology \& Software Engineering. 2(21) :207-208(2013) (In Chinese)

[3] Weize Shi. The design and implementation of railway material management system based on $B / S$ [D]. Shnaghai: School of Software Shanghai Jiaotong University(2014) (In Chinese)

[4] Peiliang Zhang. Design and implementation of material management information system for harbin railway bureau[D].Shanghai: School of Software Shanghai Jiaotong University(2012) (In Chinese)

[5] Peng-hui Zhu, Zhen-ming Zhang, Li-jiang Huang. The research of case-based reasoning in production scheduling[J].Manufacturing Automation.36(3):16-20(2014) (In Chinese)

[6] Qinling An. The principle, performance and application of Ajax [J].Network Security Technology \& Application. 12(2):23-26(2012) (In Chinese) 